\title{
La formation pratique des enseignants : le cas du système béninois
}

\section{Chronique internationale}

\section{Introduction}

"Comme dans tout système éducatif national, l'enseignant béninois reste un acteur central dans la transmission des savoirs » (UNESCO-BREDA, 2011, p. 72). Il doit disposer d'une bonne formation académique et d'une bonne formation professionnelle visant à le préparer au métier d'enseignant (UNESCO-BREDA, 2011), avec comme enjeu la capacité à dispenser des enseignements de qualité. D'où l'importance d'une formation professionnelle requise susceptible de le doter des compétences nécessaires pour délivrer un enseignement de qualité.

Au Bénin, les écoles normales d'instituteurs (ENI) assurent la formation des enseignants de la maternelle et du primaire. Les écoles normales supérieures (ENS) forment les enseignants du secondaire. Ces formations ont, l'une comme l'autre, un volet théorique et un volet pratique.

Force est de noter que depuis 2001, le secteur de l'éducation est placé sous la tutelle de quatre ministères ${ }^{1}$ qui ont connu plusieurs mutations dans leurs dénominations, leurs attributions et leur organisation (RESEN-Bénin, 2008). Avec une telle fragmentation dans la gouvernance du

1 Il s'agit en l'occurrence du ministère des Enseignements maternel et primaire (MEMP), du ministère de l'Enseignement secondaire et de la Formation technique et professionnelle (MESFTP), du ministère de l'Enseignement supérieur et de la Recherche scientifique (MESRS) et du ministère de la Culture, de l'Alphabétisation et de la Promotion des langues nationales (MCAPLN). 
système éducatif, il apparaît utile de porter un regard critique sur la formation des enseignants, plus particulièrement, la formation pratique vue comme un indicateur majeur de la qualité d'une formation professionnelle.

À partir d'une méthodologie holistique et documentaire, des entretiens auprès de cinq anciens élèves instituteurs (ENI Dogbo), de deux conseillers pédagogiques du secondaire, d'un responsable des stages et de celui de la scolarité de l'ENS (Porto-Novo), nous ambitionnons de présenter les contours du volet pratique de ces formations en mettant en relief ses particularités sans occulter les principaux défis qui pourraient en résulter.

\section{Vue d'ensemble du système béninois de formation pratique des enseignants}

Les caractéristiques du système béninois de formation pratique des enseignants peuvent se résumer, d'une part, en une gouvernance décentralisée des formations (pôle Enseignements maternel-primaire [EMP] différent du pôle Enseignement secondaire général [ESG] et Enseignement technique et de formation professionnelle [ETFP]) et, d'autre part, en une dualité de régime (formation initiale et formation continue).

\subsection{Une gouvernance décentralisée des formations}

- Les Écoles normales d'instituteurs (ENI) ( $=5)$ forment pour les enseignements maternel et primaire (EMP);

- Les Écoles normales supérieures (ENS) $(n=2)$ et l'École normale supérieure de l'enseignement technique (ENSET) $(n=1)$ forment pour l'enseignement secondaire général (ESG) et l'enseignement technique et de formation professionnelle (ETFP).

\subsubsection{Lorganisation de la formation pratique des enseignants de la maternelle et du primaire : une dualité de pôle}

\section{ENI : cheville ouvrière de la formation initiale}

Cette formation concerne des élèves instituteurs adjoints (formation au CEAP) ou des élèves instituteurs (formation au CAP) directement recrutés dans les ENI (Abomey, Dogbo, Porto-Novo, Allada, Djougou) par l'État sur concours ou sur étude de dossiers, niveau BEPC pour les premiers et niveau BAC pour les seconds. Cette formation qui se déroule dans les ENI dure deux ans. Elle fait suite à un volet théorique où les sciences cognitives, la didactique, la pédagogie générale, la pédagogie appliquée, etc. préparent l'élève au volet pratique. Ici, chaque année et pendant trois mois de la dernière année, l'élève est mis en stage dans une école. Contrairement au stagiaire en formation continue, l'élève stagiaire est placé en tutorat sous la tutelle d'un titulaire avec qui il partage la gestion de la classe durant son stage. C'est le tuteur qui assure sa formation pratique, ainsi que décrite ci-dessous au sujet de la formation continue. Son examen pratique se déroule exactement comme pour l'enseignant en formation continue. Le tableau 1 présente le synopsis de la formation pratique dans les ENI. 
Tableau 1

Synopsis des stages dans les ENI (formation initiale).

\begin{tabular}{|c|c|c|c|}
\hline Année de formation & Type de stage & Durée & Supervision \\
\hline \multirow{3}{*}{$1^{\text {re }}$ année } & Stage d'immersion & 15 jours & $\begin{array}{l}\text { Directeur d'école; Conseillers } \\
\text { pédagogiques et inspecteurs. }\end{array}$ \\
\hline & Stage d'initiation & 1 mois & $\begin{array}{l}\text { Directeur d'école; Conseillers } \\
\text { pédagogiques et inspecteurs. }\end{array}$ \\
\hline & Stage administratif & 1 semaine & $\begin{array}{l}\text { Directeur d'école; Conseillers } \\
\text { pédagogiques et inspecteurs. }\end{array}$ \\
\hline $2^{e}$ année & Stage de professionnalisation & Toute une année scolaire & $\begin{array}{l}\text { Directeur d'école; Conseillers } \\
\text { pédagogiques; responsables } \\
\text { d'unité pédagogique et } \\
\text { inspecteurs. }\end{array}$ \\
\hline
\end{tabular}

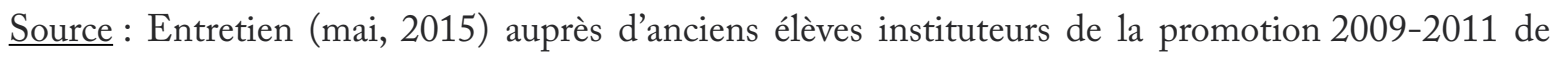
l'ENI Dogbo.

Si la typologie des stages semble adéquate, peut-on en dire autant des durées qui y sont consacrées. Elles semblent, sans contexte, courtes ou insuffisantes au regard de la grille appliquée, à ces mêmes niveaux, dans les pays du Nord. En Belgique et au Canada par exemple, elles sont plusieurs fois plus importantes, la formation faisant déjà plus de deux ans.

\section{La formation continue : une multiplicité de pôles}

La formation continue est un grand enjeu pour le système éducatif béninois. En effet, "plus de la moitié des enseignants dans l'enseignement primaire public ne disposent ni du CEAP, ni du CAP, diplômes professionnels pourtant requis pour enseigner » (UNESCO-BREDA, 2011, p. 75). Il s'agit là d'un contexte particulier dont il faudra tenir compte dans toute politique éducative au Bénin.

La formation continue des enseignants est assurée par la Direction de l'enseignement maternel (DEM), la Direction de l'enseignement primaire (DEP) et la Direction des examens et concours des enseignements maternel et primaire (DECEMP), avec l'appui de l'Institut national de formation et de recherche en éducation (INFRE).

Elle concerne des enseignants qui, ayant réussi à un concours externe du niveau BEPC, ou du niveau $\mathrm{BAC}$, doivent être formés au Certificat élémentaire d'aptitude pédagogique (CEAP), ou au Certificat d'aptitude pédagogique (CAP). Que ce soit pour le CEAP ou le CAP, les candidats réussissent d'abord à un examen théorique organisé par la DECEMP. Ce succès leur donne droit de postuler à l'examen pratique. 
Les enseignants étant déjà en situation de classe, les expériences acquises sont capitalisées et la tenue de classes est considérée comme l'équivalent du stage. Autrement dit, la formation pratique des enseignants se déroule, dans ce cas précis, alors que l'enseignant agent de l'État est titulaire depuis son recrutement, d'une classe. Pour cette formation pratique, l'enseignant reçoit l'aide des aînés et apprend sur le tas. Une note circulaire de la DECEMP précise les champs d'évaluation de l'examen pratique puis l'enseignant travaille à se mettre au pas. Le jour de l'examen pratique, il présentera une séquence de cours au niveau des différents champs à savoir l'éducation sociale (ES), l'éducation scientifique et technologique (EST), les mathématiques, le français et l'EPS. Il fera aussi l'analyse d'un des registres de classe d'une classe de même niveau, mais autre que la sienne et se prêtera à un examen pratique de législation scolaire. L'enseignant avait eu l'occasion durant sa préparation de s'exercer à toutes ces épreuves, avec l'aide des devanciers. Quelques fois, le chef de la circonscription scolaire, pour aider les candidats aux examens pratiques, organise des séances d'encadrement dans toute sa juridiction afin d'optimiser ses résultats.

\subsubsection{Lorganisation de la formation pratique des enseignants du secondaire : un souci de convergence}

L'enseignement secondaire se répartit en deux grandes unités au Bénin, à savoir l'enseignement secondaire général (ESG) et l'enseignement technique et la formation professionnelle (ETFP). Les enseignants de l'ESG sont formés par les ENS (Porto-Novo et Natitingou) au Brevet d'aptitude au professorat de l'enseignement secondaire (BAPES) ou au Certificat d'aptitude au professorat de l'enseignement secondaire (CAPES). Ceux de l'ETFP sont formés au Brevet d'aptitude au professorat de l'enseignement technique (BAPET) ou au Certificat d'aptitude au professorat de l'enseignement technique (CAPET) aujourd'hui assuré par l'École normale supérieure de l'enseignement technique (ENSET) de Lokossa. L'Institut national de l'éducation physique et sportive (INJEPS) forme quant à elle des titulaires d'un BAPES ou d'un CAPES en EPS (voir le tableau 2 ci-dessous), qui sont distribués selon les disponibilités et les besoins à l'ESG et à l'ETFP. 


\section{CHBOLIOLIIE}

Tableau 2

Synopsis de la formation pratique des enseignants d'EPS (INJEPS).

\begin{tabular}{lll}
\hline Année & Masse horaire & Supervision \\
\hline Licence 1 & 3 crédits $(25 \mathrm{~h} \times 3=75 \mathrm{~h})$ & - Inspecteurs (DIP) \\
& & - Conseillers pédagogiques \\
& & - Enseignants INJEPS \\
\hline Licence 2 & 3 crédits $(25 \mathrm{~h} \times 3=75 \mathrm{~h})$ & - Inspecteurs (DIP) \\
& & - Conseillers pédagogiques \\
& & - Enseignants INJEPS \\
\hline Licence 3 & 8 crédits $(25 \mathrm{~h} \times 8=200 \mathrm{~h})$ & - Inspecteurs (DIP) \\
& & - Conseillers pédagogiques \\
& & - Enseignants INJEPS \\
\hline Master 1 & 4 crédits $(25 \mathrm{~h} \times 4=100 \mathrm{~h})$ & - Inspecteurs (DIP) \\
& & - Conseillers pédagogiques \\
& & - Enseignants INJEPS \\
\hline Master 2 & 23 crédits $(25 \mathrm{~h} \times 23=575 \mathrm{~h})$ & - Inspecteurs (DIP) \\
& & - Conseillers pédagogiques \\
& & - Enseignants INJEPS \\
\hline
\end{tabular}

Source : Service des stages de l'INJEPS (mai 2011).

Abstraction faite de la formation des enseignants d'EPS, formation dont le volet pratique se déroule dans les établissements d'enseignement secondaire, la formation pratique des enseignants du secondaire se déroule différemment, selon que l'enseignant est en formation continue ou à plein temps. Mais, elle se déroule de la même manière, que ce soit pour un diplôme de l'enseignement technique ou un diplôme de l'enseignement général.

\section{Aspect formation continue}

Selon UNESCO-BREDA (2011, p. 75), « la grande majorité des enseignants du sous-secteur public ne disposent pas des diplômes professionnels requis pour enseigner ». Plus précisément, «la situation dans l'enseignement secondaire technique et professionnel en 2006/07 est presque similaire à celle observée dans l'enseignement secondaire général : $69 \%$ des enseignants assurent les enseignements sans les diplômes professionnels nécessaires"(UNESCO-BREDA, 2011, p. 76). C'est un constat alarmant. C'est pour remédier à la situation que la formation continue s'impose. Appréciant la situation, Dongbehounde, Guedegbe, Labe et Tchitchi (2007, p. 143) ont préféré parler de « recyclage des enseignants ». La formation continue est donc d'une grande importance dans notre contexte particulier marqué par la fermeture des établissements de formation des enseignants depuis 1987 (Agboton et Mede Moussa, 1994) qui n’ont pu être progressivement rouverts qu’à partir de 2008. 
Ainsi, l'enseignant recruté par l'État ou par le privé est en poste dans un établissement et est titulaire de plusieurs classes. Il choisit une classe du premier cycle s'il est candidat à un BAPES ou un BAPET, ou bien une classe du second cycle, s'il est candidat à un CAPES ou un CAPET. Il y sera inspecté. Il choisit alors un tuteur, un conseiller pédagogique ou un professeur certifié de sa discipline bien expérimenté, dont il communique les références à l'école. Ce dernier va suivre l'enseignant dans sa pratique de classe, en gestion de la classe, tenue des registres, organisation et compte rendu des évaluations, maîtrise des connaissances académiques, mise en œuvre pratique des stratégies d'enseignement/apprentissage. En réalité, tous ces aspects ayant fait l'objet des cours de didactique et de psychopédagogie, l'enseignant expérimenté se charge d'en assurer le transfert. Il permet au jeune enseignant d'asseoir un certain nombre de réflexes lui permettant de faire progressivement face à sa mission de professeur compétent. $\mathrm{Au}$ terme du stage, le maître apprécie le stagiaire, qui devra aussi faire face à un examen pratique d'inspection. Une appréciation chiffrée du directeur de l'établissement permet de rendre compte du rayonnement social, de l'assiduité et du sens des responsabilités du stagiaire.

\section{Aspect formation initiale}

Cette formation concerne des élèves professeurs adjoints (licence professionnelle) ou des élèves professeurs certifiés (master) recrutés par concours par les ENS. Les premiers font trois années académiques et les seconds en font cinq après le baccalauréat. Au cours de leur dernière année de formation, ces élèves sont envoyés en stage dans les établissements à second cycle pour y être placés sous la tutelle d'un conseiller pédagogique ou d'un professeur certifié bien expérimenté. Le stagiaire, dans ce cas précis, n'est pas titulaire de la classe, mais y travaille sous la direction du tuteur. Le tuteur assure sa formation pratique comme indiqué plus haut. Ainsi, comme pour l'enseignant en formation continue, le stagiaire sera apprécié de son tuteur, du directeur de l'établissement et fera face à une inspection. Le tableau 3 résume la situation des stages ici.

Tableau 3

Synopsis des stages en formation initiale dans les ENS.

\begin{tabular}{clcl}
\hline Type de stage & Période & Masse horaire & Évaluation \\
\hline Stage d'initiation & Fin de la $2^{\mathrm{e}}$ année & 2 crédits & Inspection de qualification \\
& Semestre 4 & $25 \mathrm{~h} \times 2=50 \mathrm{~h}$ & pédagogique \\
& & & \\
\hline $\begin{array}{c}\text { Stage de qualifi- } \\
\text { cation }\end{array}$ & Fin de la $3^{\mathrm{e}}$ année & 6 crédits & Inspection de qualification \\
\cline { 2 - 3 } & Semestre 6 & $25 \mathrm{~h} \times 6=150 \mathrm{~h}$ & pédagogique \\
\hline
\end{tabular}

$\underline{\text { Source }}$ : Entretien (mai 2015) avec le responsable des stages et le chef scolarité de l'ENS (PortoNovo). 


\section{La formation pratique des enseignants dans le système éducatif béninois : des défis immenses}

La formation pratique des enseignants ainsi décrite appelle quelques questionnements sur sa qualité. En effet, on peut souligner des défis quantitatifs et qualitatifs aussi bien en formation initiale qu'en formation continue. Des extraits du méta-rapport au titre significatif : La question enseignante au Bénin : un diagnostic holistique pour la construction d'une politique enseignante consensuelle, soutenable et durable, réalisé par UNESCO-BREDA en 2011, serviront ici de points d'ancrage.

\subsection{D'importants défis quantitatifs}

Il s'agit avant tout de porter un regard critique sur la capacité physique des établissements de formation (ENI et ENS) en rapport avec les besoins réels du système éducatif.

Il apparaît que la capacité globale de formation initiale est de l'ordre de 300 places pour les enseignants de maternelle. Il en est de même pour chacune des ENI dédiées à la formation initiale des enseignants du primaire, ce qui représente une capacité globale de 1500 places pour l'ensemble des 5 ENI dédiées au primaire. Cependant, les effectifs d'élèves-maîtres en formation au cours de l'année scolaire 2009/10 dépassent largement ces capacités physiques normales, ce qui pourrait traduire de mauvaises conditions d'encadrement. (UNESCOBREDA, 2011, p. 79)

C'est dire donc qu'il y a lieu, au regard du ratio capacité d'accueil et effectifs en formation, de craindre ou de présumer de mauvaises conditions de formation, notamment au niveau des stages qui exigent un encadrement plus soutenu, voire personnalisé. Le tableau 4 suivant offre une vue panoramique des mauvaises conditions d'encadrement.

Tableau 4

Capacité physique et besoins annuels de formation initiale pour les enseignants du sous-secteur public.

\begin{tabular}{|c|c|c|c|c|c|c|}
\hline $\begin{array}{c}\text { Nivesu } \\
\text { densurigaremeat }\end{array}$ & 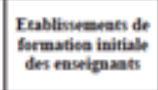 & $\begin{array}{l}\text { Capacitis } \\
\text { physiques } \\
\text { normales }\end{array}$ & 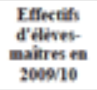 & 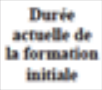 & $\begin{array}{c}\text { Flux } \\
\text { samuel } \\
\text { woyea de } \\
\text { sortie }\end{array}$ & 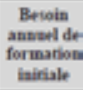 \\
\hline Maxtenel & "01 EN & 300 & 347 & $2 \mathrm{ams}$ & 174 & 258 \\
\hline Riminite & 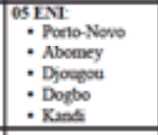 & $\begin{array}{c}300 \times 5= \\
1500\end{array}$ & 2157 & $2 \mathrm{ams}$ & $10 \pi 0$ & 2107 \\
\hline 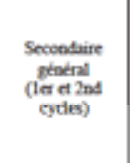 & 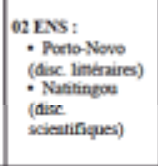 & $\begin{array}{c}225 \\
\text { (ENS } \\
\text { Namingou } \\
\text { sonte) }\end{array}$ & 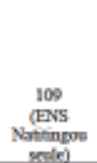 & $3 \mathrm{ass}$ & 75 & 2200 \\
\hline
\end{tabular}

Source : UNESCO-BREDA (2011, p. 79). 
Le tableau montre très clairement qu'aussi bien à la maternelle, au primaire qu'au secondaire, le nombre d'enseignants en formation pulvérise les capacités d'accueil des établissements. On en déduit que les capacités physiques actuelles des établissements de formation sont nettement insuffisantes par rapport aux besoins de formation. Un autre problème non moins important se rapporte au nombre d'enseignants formés au regard des besoins réels du pays. Ainsi, normalement, au niveau des deux ENS, le nombre total d'enseignants formés chaque année devrait être de 150, ce qui demeure encore très loin des besoins.

\subsection{D'importants défis qualitatifs}

Nous nous référons ici à la qualité de la formation, initiale ou continue. Disons sans ambages qu'en dépit des efforts pour atteindre cet objectif, bien des insuffisances persistent. D'abord, le profil des candidats recrutés pour la formation suscite des interrogations.

En effet, après avoir échoué au concours d'entrée, les candidats qui pourraient supporter financièrement la formation n'hésitent pas [à] demander une admission à titre payant. Ces derniers sont parfois acceptés sur étude de dossiers en fonction des places disponibles. Ils paient donc $170000 \mathrm{FCFA}$ pour les titulaires du BEPC et $190000 \mathrm{FCFA}$ pour les titulaires du BAC sur les deux ans de formations. Cependant, du fait qu'ils aient échoué au concours d'entrée, il y a lieu de s'interroger si le niveau de ces candidats repêchés leur permet de pouvoir suivre la formation. Cette question mérite toute son attention au regard du nombre de candidats admis à titre payant qui devient de plus en plus important que le nombre de candidats admis sur concours. Par exemple, les candidats admis à titre payant au cours de l'année scolaire 2009-10 représentaient près de $55 \%$ des effectifs des élèvesmaîtres pour l'ensemble des six ENI, alors qu'ils ne représentaient que 38 \% en 2008-09. (UNESCO-BREDA, 2011, p. 81).

Aussi peut-on se demander si le niveau académique d'entrée est le plus adapté pour suivre cette formation? Si les deux épreuves du concours sont-elles les plus appropriées pour sélectionner le profil adéquat à la formation? Ne serait-il pas plus bénéfique de considérer un niveau académique plus élevé pour la maternelle et le primaire en supprimant les BEPC pour considérer uniquement le BAC?

De même, il n'est pas superflu de se demander si la durée des deux ans de formation dans les ENI est optimale au regard du contenu de la formation.

Finalement, sur l'ensemble des deux ans de formation, les dimensions académique, professionnelle et pratique semblent être bien prises en compte. Il reste la difficile question de l'articulation entre les différentes dimensions, mais aussi du lien avec les compétences désirées à la sortie. Au regard du contenu de la formation et des volumes horaires correspondant, les cours théoriques occupent 660 heures, alors que les stages pratiques comptent pour 264 heures la première année et occupent toute la seconde année. On pourrait se poser la question de savoir s'il s'agit là du meilleur dosage entre la théorie et la pratique? (UNESCO-BREDA, 2011, p. 82). 
En ce qui concerne la formation continue, l'on gagnerait, dans notre contexte particulier, à la restructurer afin d'en faire un puissant outil de requalification professionnelle des enseignants au service d'un système éducatif performant, efficace et efficient. Pour ce faire, il faut commencer par mettre un thème à sa fragmentation, tant en ce qui concerne les instances d'organisation des formations que du contenu de ces mêmes formations. (UNESCO-BREDA, 2011, p. 92).

La formation continue ne peut plus être un événement ponctuel de quelques jours ou des vacances scolaires avec plusieurs acteurs et auquel les enseignants sont conviés selon la disponibilité de salles de classes laissées vacantes et du personnel pédagogique utilisée par ailleurs. Il est nécessaire de s'orienter vers un degré d'organisation comparable à ce qu'on observe pour la formation initiale. Il ne s'agit pas uniquement de questions de durée ou de période de formation; les contenus, les modalités d'organisation, la question de l'évaluation et la conception même de la formation continue sont à considérer. Le dispositif doit in fine permettre de former les enseignants sans la formation initiale requise, d'assurer l'articulation entre la formation initiale et la formation continue de manière à permettre aux enseignants formés de revenir souvent se reformer dans le cadre de la formation continue, mais aussi de servir de leviers pour le développement professionnel des enseignants. (UNESCO-BREDA, 2011, p. 97)

Au regard de ce rapide tour d'horizon de la formation pratique des enseignants au Bénin, il en ressort que des efforts importants sont déployés pour doter le système éducatif d'enseignants qualifiés et professionnels. Toutefois, force est de constater des insuffisances çà et là qui semblent encore l'éloigner de cet objectif. Ces difficultés, comme montrées ici, se situent tant au plan quantitatif que qualitatif aussi bien au niveau de la formation initiale que de la formation continue.

\section{Conclusion}

L'objectif de l'article était de présenter la formation pratique des enseignants dans le système éducatif béninois à travers ses forces et ses faiblesses. Sans prétendre à l'exhaustivité, nous avons dressé un portrait réel de ladite formation dans notre contexte particulier. Au terme de cet exercice passionnant, il est à retenir le souci des autorités responsables de l'éducation de former des enseignants compétents et aptes à remplir convenablement leur mission. Toutefois, à l'instar de la plupart des États en voie de développement, l'État béninois n'arrive pas encore à asseoir un système ou un dispositif de formation des enseignants et notamment de formation pratique des enseignants digne de sa renommée de quartier latin de l'Afrique, encore moins, de ses ambitions pour la réussite de l'Éducation pour tous. De la qualité de la formation des enseignants dépendra, entre autres, la qualité du système éducatif et, partant, des produits de l'école. Par conséquent, il urge que l'accroissement des capacités physiques des établissements de formation des enseignants soit hissé au rang de priorité nationale afin de faire correspondre les effectifs des formés avec les besoins réels de l'école. De même, il est impératif de repenser le profil des candidats dans les ENI et ENS, la procédure, la durée, le contenu de la formation avec un meilleur dosage de la formation pratique par rapport à la formation théorique. Enfin, la formation continue mérite de toute urgence une restructuration pour plus de symphonie, mais surtout pour devenir un véritable instrument de requalification professionnelle dans la carrière de l'enseignant. 


\section{Références}

Agboton, S. et Mede Moussa, Y. (1994). Formation des enseignants dans une perspective d'éducation permanente au Bénin. International Review of Education, 40(3), 223-234. http://dx.doi.org/10.1007/bf01257777

Bogdan, R. et Biklen, S. K. (1992). Qualitative research for education : an introduction to theory and methods ( ${ }^{\mathrm{e}}$ éd.). Boston, MA : Allyn and Bacon.

Dongbehounde, J., Guedegbe, R., Labe, F. et Tchitchi, T. (2007). Les politiques nationales de formation, de recrutement et de rétention des enseignants à tous les niveaux du système éducatif en Afrique : la situation en République du Bénin. Dans T. Karsenti, R.-P. Garry, J. Bechoux et S. Tchameni Ngamo (2007), La formation des enseignants dans la francophonie : diversités, défis et stratégies d'action (p. 137-154). Montréal : AUF.

Dossat, L. et Sfeir, Y. (2009). Dispositif de qualification des enseignants contractuels locaux reversés dans la Fonction publique au Bénin (Rapport de mission du 27 mai au 2 juin 2010 à Cotonou).

Gouvernement du Bénin. (2006). Plan décennal de développement du secteur de l'éducation 2006-2015. Repéré à http://planipolis.iiep.unesco.org/upload/Benin/Benin PDDE T1 T2 CMEF.pdf

RESEN-Bénin. (2008). Le système éducatif béninois : Analyse sectorielle pour une politique éducative plus équilibrée et plus efficace. Repéré à http://www-wds.worldbank.org/external/default/WDSContentServer/WDSP/ IB/2009/06/04/000020953 20090604153805/Rendered/PDF/486030PUB0FREN101Official0Use0Only1.pdf

Somekh, B. et Lewin, C. (2005). Research methods in the social sciences. Londres : Sage.

UNESCO. (2010). Guide méthodologique d'analyse de la question enseignante. Repéré à http://unesdoc.unesco.org/images/0019/001901/190129f.pdf

UNESCO-BREDA. (2009). La scolarisation primaire universelle en Afrique : le défi enseignant. Repéré à http://www.uis.unesco.org/Library/Documents/scolarisation-primaire-universelle-afrique-defi-enseignants-2009-fr.pdf

UNESCO-BREDA. (2011). La question enseignante au Bénin : un diagnostic holistique pour la construction d'une politique enseignante consensuelle, soutenable et durable. Repéré à http://unesdoc.unesco.org/images/0021/002171/217150f.pdf 